\title{
Evaluación de la motivación académica y la ansiedad escolar y posibles relaciones entre ellas
}

\author{
Debora CeilioFemandes- UniversidadedbValedoSapucaí, PausoAlege, Minas Gerais, Brasil \\ Mariana Alves Silveira - UniversidadedoValedoSapuraí, PausoAleege, Minas Gerais, Brasil
}

\begin{abstract}
Resumen
La motivación académica y la ansiedad en el contexto académico son factores determinantes en la vida del alumno, tanto desde el punto de vista emocional como de rendimiento. El objeto de este estudio fue evaluar la motivación y la ansiedad académica en estudiantes y realizar análisis de correlación y regresión entre la motivación académica y la ansiedad escolar. Los niños obtuvieron puntuaciones bajas de ansiedad y moderadas de motivación general, niveles por encima del promedio de motivación extrínseca y por debajo del promedio de motivación intrínseca. El análisis de correlación indicó que todas las correlaciones fueron significativas y que todas fueron positivas, excepto las correlaciones entre la ansiedad y la motivación intrínseca y entre la motivación intrínseca y extrínseca, que fueron negativas. El análisis de regresión reveló que la motivación extrínseca explica una parte significativa de la ansiedad. Esos datos elucidan la relación existente entre los dos tipos de motivación y la ansiedad escolar.

Palabrasdave motivación académica; motivación extrínseca; motivación intrínseca; ansiedad escolar.
\end{abstract}

Assessment of academic motivation and school anxiety and possible relationship between them

\begin{abstract}
Academic motivation and anxiety in the academic context are both determinant factors in the student's life, from the emotional and achievement points of view. The aim of this study was to assess motivation and school anxiety of students and to carry out correlation and regression analysis between academic motivation and school anxiety. Children obtained low score of anxiety and moderate score of general motivation, above average levels of extrinsic motivation and under mean levels of intrinsic motivation. Correlation analysis indicated that all correlations were significant and that all of them were positive, except the correlation between anxiety and intrinsic motivation and between intrinsic and extrinsic motivation, which were negative. Regression analysis revealed that extrinsic motivation explains a significant part of anxiety. The data elucidates the existent relation between both types of motivation and school anxiety.
\end{abstract}

Keguards academic motivation; extrinsic motivation; intrinsic motivation; school anxiety.

\section{Avaliação da motivação acadêmica e ansiedade escolar e possível relação entre elas}

\begin{abstract}
Resumo
A motivação acadêmica e a ansiedade no contexto acadêmico são fatores determinantes na vida do aluno, tanto do ponto de vista emocional como do rendimento. 0 objetivo deste estudo foi avaliar a motivação e a ansiedade acadêmica em estudantes e realizar análise de correlação e regressão entre a motivação acadêmica e a ansiedade escolar. As crianças obtiveram pontuações baixas de ansiedade e moderadas de motivação geral, níveis acima da média de motivação extrínseca e abaixo da média de motivação intrínseca. A análise de correlação indicou que todas as correlações foram significativas e que todas foram positivas, com exceção das correlações entre a ansiedade e a motivação intrínseca e entre a motivação intrínseca e extrínseca, que foram negativas. A análise de regressão revelou que a motivação extrínseca explica uma parte significativa da ansiedade. Esses dados elucidam a relação existente entre os dois tipos de motivação e a ansiedade escolar.
\end{abstract}

Palavasdhave motivação acadêmica; motivação extrínseca; motivação intrínseca; ansiedade escolar.

El aula es un contexto complejo, en el cual interactúan diversas variables que pueden llevar a una facilitación 0 a un incremento de la dificultad del proceso de aprendizaje de los alumnos. D ebido a la complejidad del ser humano y de las interacciones humanas, la comprensión de parte de la realidad de la sala de aula es posible cuando se delimitan variables específicas como objeto de estudio. De ese modo, tanto la motivación como la ansiedad son variables que influyen sobre el comportamiento de los alumnos en el contexto educacional y en el proceso de aprendizaje y, por ello, se necesitan más estudios de ambos constructos en ese contexto específico.

Respecto a la motivación, son escasas las investigaciones cuantitativas y los datos empíricos sobre su relevancia en el contexto escolar (Martinelli \&
Sisto, 2011). La motivación no es una variable observable, sino un constructo hipotético que parte de inferencias realizadas a partir de manifestaciones de la conducta. Ella puede comprenderse como una fuerza interna que emerge, regula y sostiene las acciones (Vemon, 1973) o como aquello que mueve a una persona, la hace actuar o cambiar de sentido (Bzuneck, 2000). La motivación es uno de los factores que lleva al alumno a la escuela, le instiga y le hace empezar una conducta hacia un objetivo. También garantiza la persistencia, pues durante el proceso surgen obstáculos y situaciones de fracaso que pueden hacer el sujeto evaluar sus conceptos y cambiar su conducta (Bzuneck, 2000).

Los enfoques contemporáneos de la motivación, dentro de los cuales está la teoría sociocognitiva, 
consideran que la motivación puede ser intrínseca y extrínseca, en función del tipo de meta que debe alcanzarse (D eci \& Ryan, 2000). De ese modo, cuando una acción se regula por intereses propios o cuando depende de factores externos, la claridad de la acción y el planeamiento difieren mucho (Martinelli \& Sisto, 2011). Así pues, la motivación intrínseca se refiere a la realización de una actividad por el placer o satisfacción de participar en la actividad (Deci \& Ryan, 2000). Las necesidades intrínsecas del ser humano, 0 más concretamente del alumno, que influyen sobre la motivación intrínseca son la autonomía, la competencia y el establecimiento de vínculos. Esas tres necesidades están integradas y son interdependientes y, por ello, fortalecer una de ellas repercute sobe las demás (D eci \& Ryan, 2000).

Además, el ser humano también se influye por el contexto y su ambiente social, lo que significa que asimismo debe responder a las presiones y demandas sociales y mostrar su valor. Así, ese tipo de motivación se considera extrínseca a la tarea o la actividad. Sería el caso de obtener recompensas materiales o sociales, reconocimiento social y evitar punición (Fortier, Vallerand \& Guay, 1995). En base a eso, hay autores que afirman que ambos tipos de motivación, la intrínseca y la extrínseca, son fundamentales para el buen rendimiento académico y para la persistencia escolar (Pintrich, 2003).

En ese sentido, la investigación llevada a cabo por Martinelli y Sisto (2010) mostró que existe una reducción gradual de la motivación intrínseca y extrínseca conforme los alumnos avanzan en los cursos escolares. Esos datos se obtuvieron a partir de un análisis de varianza que reveló que las diferencias en la motivación de los alumnos en función del curso no se deben al acaso, tanto en lo que respecta a la motivación intrínseca $(\mathrm{F}=5,775 ; \mathrm{p}=0,003)$ como a la motivación extrínseca $(\mathrm{F}=24,927 ; \mathrm{p}<0,001)$.

Posteriormente, se llevó a cabo la prueba de Tukey. En el caso de la motivación intrínseca, se indicó que los alumnos del tercer curso se diferenciaron significativamente de los de cuarto y quinto $(\mathrm{p}=0,05)$. La media de las puntuaciones de los alumnos del tercer curso fue 18,08, mientras que las medias de los alumnos del cuarto y quinto curso fueron, respectivamente, 17,25 y 17,38. Además, no hubo diferencia significativa entre las medias de los alumnos del cuarto y del quinto curso $(\mathrm{p}=0,89)$.

En lo que respecta a la motivación extrínseca, el análisis de Tukey reveló que los niños del tercer curso presentaron una motivación extrínseca significativamente superior a la de los niños de cuarto y quinto $(\mathrm{p}=0,05)$, siendo que éstos últimos no se diferenciaron entre sí $(\mathrm{p}=0,22)$. En ese sentido, la media de las puntuaciones del tercer curso fue 10,92, mientras que las medias de los alumnos de cuarto y quinto cursos fueron 8,44 y 7,49, respectivamente. Por lo tanto, se observó una reducción de las motivaciones intrínseca y extrínseca de los alumnos desde el tercero al quinto curso.

Guay, Ratelle y Chanal (2008) realizaron una revisión de los estudios relacionados con la motivación. La revisión indicó que cada tipo de motivación influye sobre el comportamiento y el rendimiento de las personas y los alumnos de maneras diferentes. Por ejemplo, los autores mencionan estudios que indican que los alumnos que presentan un tipo de motivación más autónoma (intrínseca) son también más persistentes, ya que hay trabajos que muestran que alumnos que abandonaron la escuela presentaban menos motivación intrínseca en comparación con los alumnos que persistían (Vallerand, Fortier \& Guay, 1997). La revisión también indicó que los estudiantes con motivación autónoma (intrínseca) relatan más emociones positivas en el aula, más placer con el trabajo académico y más satisfacción con la escuela. Por el contrario, Ryan y Connell (1989) mostraron que la motivación extrínseca presenta una correlación baja y positiva con la ansiedad cognitiva en los niños $(r=0,22$; $\mathrm{p}<0,01)$, al igual que la motivación intrínseca $(\mathrm{r}=0,21$; $p<0,5)$.

La investigación llevada a cabo por Gottfried (1985) estableció una importante relación entre motivación y ansiedad. En uno de sus estudios, la autora evaluó la motivación académica intrínseca y la ansiedad académica de 166 niños para diferentes áreas, como la lectura, las matemáticas, estudios sociales, ciencias y motivación general. Se observaron correlaciones bajas y moderadas y negativas entre la motivación intrínseca y la ansiedad académica en todas las áreas, con valores de correlación comprendidos entre $-0,38$ y $-0,52 \quad(\mathrm{p}<0,001)$. Por ejemplo, la correlación entre la motivación intrínseca orientada a la lectura y la ansiedad académica relacionada con lectura fue -0,47. Eso indica que cuánto más motivados intrínsecamente los alumnos en relación a la lectura, menor será la manifestación de la ansiedad relacionada con la lectura. Se encontró ese mismo patrón de resultado para todas las áreas y para la motivación intrínseca general. Cabe señalar que los resultados de esa investigación discrepan de los resultados de Ryan y Connell (1989) respecto a la motivación intrínseca y la ansiedad.

Las relaciones observadas entre los dos tipos de motivación y la ansiedad requieren estudios más exhaustivos, pues la ansiedad cuando se instala 
desproporcionadamente es un factor que perjudica el desarrollo social y cognitivo de los niños. Por ejemplo, alumnos más ansiosos pueden presentar más dificultad para concentrarse y mantener la concentración, memorizar, organizar informaciones etc. Además, si su intensidad alcanza niveles patológicos, puede manifestarse como una experiencia de miedo, terror, alarme, pánico, entre otras. Personas con altos niveles de ansiedad presentan una sensación implícita de peligro inminente sin riesgo real, acompañada de sensación de incomodidad corporal. También se pueden observar manifestaciones corporales tales como sudores, temblor, nausea, vomito, palpitaciones y dolores abdominales (Andrade \& Gorenstein, 1998).

En lo que atañe a la diferencia de niveles de ansiedad entre chicos y chicas, tradicionalmente, se han detectado niveles más altos de ansiedad general en chicas que en chicos (Silverman, La Greca \& Wassertein, 1995; Singh, Moraes \& Ambrosano, 2000; Batista \& O liveira, 2005). No obstante, no está claro si dichas diferencias se encuentran también en el contexto escolar.

A ese respecto, es interesante comentar el estudio llevado a cabo por Isolan, Salum, Osowski, Amaro y Manfro (2011), en el cual se aplicó la escala de ansiedad general SCARED en una amplia muestra de 2410 jóvenes. Se relató que las chicas presentaron más síntomas de ansiedad que los chicos en las subescalas y en la escala general (Isolan \& cols., 2011). Los autores relataron también que los síntomas de ansiedad de los jóvenes brasileños oscilaron de moderado a alto, representando valores superiores a los niveles observados en otras poblaciones.

En lo referente a las investigaciones de la ansiedad y la motivación de forma conjunta, son escasos los trabajos que investigan ambos constructos, pero Urhahne Chao, Florineth, Luttenberger y Paechter (2011) investigaron la influencia del juicio de los profesores respecto a sus alumnos. Los resultados mostraron que los profesores sobreestiman la mayoría de sus alumnos y subestiman la minoría. Lo más interesante de este estudio fue la comparación entre alumnos sobreestimados y subestimados; la puntuación en un examen de matemáticas de ambos grupos fue similar, no hubo diferencias significativas en relación con el nivel de aspiración y motivación para aprender, sin embargo los autores observaron que los estudiantes subestimados presentaron valores inferiores en la expectación de éxito y el autoconcepto académico. Además, se obtuvo una intensidad de ansiedad en situación de evaluación superior para el grupo subestimado (Urhahne \& cols., 2011), sugiriendo que pese a que los alumnos de los dos grupos presenten rendimiento similar en la evaluación, el grupo subestimado presentó un nivel superior de ansiedad, lo que supone un obstáculo que debe ser superado para la obtención del rendimiento académico observado.

Llevando en consideración la literatura especializada de la ansiedad y la motivación y la insuficiencia de investigaciones que abarcan ambos constructos, teniendo en cuenta específicamente el contexto escolar, el objeto de este estudio fue evaluar la ansiedad escolar y la motivación académica en niños y averiguar la posible relación entre la motivación intrínseca, extrínseca y la ansiedad en el contexto educacional.

\section{Partiapantes}

\section{Método}

La muestra se compuso de 195 alumnos de segundo al quinto curso de la Enseñanza Fundamental (muestra por conveniencia) de una escuela privada del sur de Minas Gerais, Brasil. Los participantes presentaron consentimiento informado firmado por uno de los padres o responsable. En la Tabla 1 se exhiben las informaciones relativas a las características de los sujetos en función del curso, edad y sexo.

Tabla 1. D istribución por curso, edad y sexo de los participantes

\begin{tabular}{|c|c|c|c|c|c|c|c|c|}
\hline \multirow{3}{*}{ Edad } & \multicolumn{8}{|c|}{ Curso } \\
\hline & \multicolumn{2}{|c|}{$2^{\underline{0}}$} & \multicolumn{2}{|c|}{$3^{0}$} & \multicolumn{2}{|c|}{$4^{0}$} & \multicolumn{2}{|c|}{$5^{0}$} \\
\hline & $\mathrm{F}$ & $\mathrm{M}$ & $\mathrm{F}$ & $\bar{M}$ & $\mathrm{~F}$ & $\mathrm{M}$ & $\bar{F}$ & $\mathrm{M}$ \\
\hline 7 & 15 & 9 & 0 & 0 & 0 & 0 & 0 & 0 \\
\hline 8 & 12 & 5 & 17 & 23 & 0 & 1 & 0 & 0 \\
\hline 9 & 0 & 0 & 7 & 10 & 20 & 20 & 0 & 0 \\
\hline 10 & 0 & 0 & 0 & 0 & 8 & 6 & 13 & 15 \\
\hline 11 & 0 & 0 & 0 & 0 & 0 & 0 & 9 & 5 \\
\hline Total & 27 & 14 & 24 & 33 & 28 & 27 & 22 & 20 \\
\hline
\end{tabular}

En la Tabla 1 se visualiza que los niños presentaban edades comprendidas entre 7 y 11 años.
La muestra se compuso de 27 niñas del segundo curso, 24 del tercero, 28 del cuarto y 22 del quinto, 
totalizando 101 niñas. En el segundo curso, participaron 14 niños, en el tercero, 33 y en cuarto y quinto curso participaron 27 y 20, respectivamente, totalizando 94 niños.

\section{Mateiales}

Inventario de Ansiedad Escolar (Oliveira \& Sisto, 2002)

La versión actual del inventario se compone de 38 ítems en formato de escala Liket con tres opciones de respuesta, en las cuales el sujeto indica la frecuencia en la que ocurren las frases de los ítems (siempre, a veces, nunca). De los 38 ítems, ocho de ellos son de compensación, porque contienen frases con sentido positivo para contrarrestar el sentido negativo de las frases relacionadas con la ansiedad de los demás ítems. Por ello, esos ítems no se suman para la obtención de la puntuación de la ansiedad.

Las puntuaciones de la escala varían desde 0 a 60 puntos. Los ítems están acordes con las definiciones para el diagnóstico del trastorno de ansiedad en la escuela aceptadas pela comunidad científica internacional, es decir, la Clasificación Internacional de Enfermedades (CIE) y el Manual de Diagnóstico y Estadística (DSM) de la Asociación Norte-Americana de Psiquiatría. El desarrollo de la escala se basó en el análisis factorial llevado a cabo por Oliveira (2001) y el inventario posee propiedades psicométricas adecuadas para su utilización, tales como validez de constructo e indicadores de fiabilidad adecuados (Oliveira, 2001). La escala fue validada para alumnos de cuarto y quinto curso con edades comprendidas entre 9 y 12 años (Oliveira, 2011).

Escala para Evaluación de la Motivación Escolar Infanto-juvenil (EAME-IJ, Martinelli \& Sisto, 2011)

La escala se construyó según los supuestos de la teoría de autodeterminación y propone la evaluación de las motivaciones intrínsecas y extrínsecas. La escala contiene 20 ítems, 10 referentes a cada tipo de motivación. Se elaboró la escala considerando los resultados del análisis factorial y del análisis mediante el modelo de Rasch (1960) de las opciones de respuesta de la escala Liket. Las puntuaciones de la escala general van de 0 a 40 puntos y las de las escalas específicas van de cero a 20 puntos. Su validación incluye personas de 8 a 11 años, presenta propiedades psicométricas adecuadas que justifican su uso y viene siendo perfeccionada con regularidad. Son ejemplos de ítems las frases "Yo estudio solamente para aprobar" y "Yo me siento feliz cuando veo que estoy mejorando en la escuela".

\section{Procedimientos}

\section{Consideraciones éticas}

En primer lugar, se obtuvo la autorización de la dirección del colegio en el que se recogieron los datos. Posteriormente, se envió el proyecto al Comité de Ética de la Universidad (Univás) y tras su aprobación (Protocolo no 1737/11) se planeó las fechas para recoger los datos de modo a no perjudicar la rutina de la escuela.

Recogida de datos

Las aplicaciones se realizaron en la escuela, de forma colectiva y en las aulas. Las investigadoras instruyeron los alumnos sobre cómo marcar las opciones de respuesta y en el caso de los alumnos del segundo curso, las investigadoras leyeron en voz alta las frases de las escalas. El tiempo de respuesta de las dos escalas fue aproximadamente de 25 minutos y los alumnos no presentaron dificultades para contestarlas.

\section{Resultados}

Los resultados de la evaluación de la ansiedad, motivación, correlación y regresión se presentan a continuación. Inicialmente, en la Tabla 2 , se muestran las medias de la puntuación de los participantes en las escalas de ansiedad y motivación, en función del sexo. Se exhiben también los promedios de la motivación intrínseca y extrínseca.

Tabla 2. Media y DT de los sexos en ansiedad, motivación general, intrínseca e extrínseca

\begin{tabular}{lcccccccc}
\hline \multirow{2}{*}{ Sexos } & \multicolumn{2}{c}{ Ansiedad } & \multicolumn{6}{c}{ Motivación } \\
\cline { 2 - 8 } & Media & DT & Media & DT & Media & DT & Media & DT \\
\hline Femenino & 21,88 & 8,84 & 22,43 & 4,30 & 16,80 & 2,86 & 5,64 & 4,07 \\
Masculino & 23,22 & 9,69 & 23,35 & 6,40 & 14,65 & 4,48 & 8,70 & 5,65 \\
General & 22,53 & 9,27 & 22,88 & 5,42 & 15,75 & 3,9 & 7,12 & 5,12 \\
\hline
\end{tabular}

Se observa que las puntuaciones de ansiedad están por debajo del promedio, mientras que las puntuaciones de la motivación intrínseca están por encima de la media y las de la motivación extrínseca son inferiores a la media. Para averiguar si las diferencias entre las medias de los chicos y las chicas presentadas en la Tabla 2 son significativas, se realizó la comparación entre las medias por la prueba $t$ de 
Student. En el caso de la Escala de Ansiedad, no se obtuvo diferencia significativa entre los grupos, pues $\mathrm{t}=-1,01(\mathrm{~g}=193 ; \mathrm{p}=0,313)$. Para la escala de motivación, la motivación general no produjo diferencias significativas entre los promedios. La comparación reveló que $\mathrm{t}=1,18$, que gl fue 193 y p se correspondió a 0,24 . No obstante, la motivación intrínseca produjo un valor de $t=4,01, g=193$ y $p<0,001$, lo que indica que las chicas mostraron una motivación intrínseca significativamente superior a la de los chicos. El tamaño del efecto de la diferencia fue $\mathrm{d}=0,63$, considerado moderado. Finalmente, la motivación extrínseca fue significativamente superior para los chicos, puesto que $\mathrm{t}=-4,36, \mathrm{~g}=193$ y $p<0,001$. El tamaño del efecto de la diferencia, en ese caso, fue d $=0,66$, también considerado moderado. En relación con las diferencias entre los cursos, se llevó a cabo un ANOVA con la prueba a posteriori de Tukey. En la escala de ansiedad se observó la formación de solamente un grupo, con pigual a 0,52. En lo que se refiere a la motivación, tampoco hubo diferencia significativa entre los cursos. El valor de p fue de 0,22, indicando que las diferencias entre las medias se deben al azar. No obstante, las subescalas de motivación intrínseca y extrínseca presentaron diferencias significativas, como puede visualizarse en la Tabla 3.

Tabla 3. Agrupamientos de la prueba de Tukey en la escala de motivación intrínseca en función del curso

\begin{tabular}{lccc}
\hline Curso & $\mathrm{N}$ & 1 & 2 \\
\hline 3 & 57 & 14,88 & 15,38 \\
4 & 55 & 15,38 & 16,31 \\
5 & 42 & 16,31 & 16,93 \\
2 & 41 & & 0,20 \\
\hline $\mathrm{F}$ & & 0,26 & \\
\hline
\end{tabular}

Se observa, en la Tabla 3, que el tercer curso está localizado en el grupo 1, que el segundo curso está en la segunda agrupación y que los demás cursos están en ambos grupos. El tamaño del efecto de las diferencias encontradas fue $\mathrm{f}=0,83$, lo que indica que el tamaño de la diferencia es grande. En el caso de la motivación extrínseca, la Tabla 4 muestra la formación de dos grupos en función de los cursos.

Tabla 4. Subconjuntos formados por la prueba de Tukey en función de los cursos en la motivación extrínseca

\begin{tabular}{lccc}
\hline Curso & $\mathrm{N}$ & 1 & 2 \\
\hline 5 & 42 & 5,57 & \\
4 & 55 & 6,45 & 6,45 \\
2 & 41 & 6,88 & 6,88 \\
3 & 57 & & 9,07 \\
\hline $\mathrm{F}$ & & 0,58 & 0,05 \\
\hline
\end{tabular}

La Tabla 4 muestra que el quinto curso está presente en el primer agrupamiento, mientras que el tercer curso se localiza solamente en el segundo grupo. Los demás cursos se encuentran en los dos subconjuntos formados. El tamaño del efecto de las diferencias fue $\mathrm{f}=1,55$, indicando que la diferencia es grande. El estudio de correlación entre las puntuaciones de las dos escalas puede verse en la Tabla 5. Se visualizan los valores de correlación entre la ansiedad, motivación general, motivación intrínseca y extrínseca.

Tabla 5. Correlación entre ansiedad, motivación general, intrínseca y extrínseca

\begin{tabular}{|c|c|c|c|c|}
\hline & & MotivaciónG & MotivaciónIN & MotivaciónEX \\
\hline Ansiedad & Correlación de Pearson & $0,26^{* *}$ & $-0,19 * *$ & $0,41^{* *}$ \\
\hline MotivaciónG & Correlación de Pearson & & $0,43^{* *}$ & $0,73^{* *}$ \\
\hline MotivaciónIN & Correlación de Pearson & & & $-0,30 * *$ \\
\hline & $\mathrm{r}$ (bilateral) & & & 0,000 \\
\hline
\end{tabular}

La correlación es significativa a 0,01

Se observa en la Tabla 5 que todas las correlaciones fueron significativas. La correlación entre la ansiedad y la motivación general fue positiva y baja, la correlación entre la ansiedad y la motivación intrínseca fue negativa y baja y entre la ansiedad y la motivación extrínseca fue positiva y moderada. A su 
vez, la correlación entre la motivación general y la intrínseca fue positiva y moderada y entre la general y la extrínseca fue positiva y alta. Finalmente, la correlación entre la motivación intrínseca y la extrínseca fue negativa y moderada.

Se realizó un análisis de regresión múltiple entre las variables estudiadas, mediante el método por pasos (stepvise). Se estableció la ansiedad como variable dependiente y la motivación general, intrínseca y extrínseca como variables independientes 0 explicativas. La única variable independiente que alcanzó la significación estadística para explicar el nivel de ansiedad fue la motivación extrínseca ( $p<0,001)$, conforme se visualiza en la Tabla 6. La motivación general y la intrínseca presentaron valores de piguales a 0,279, no alcanzando el nivel de significancia.

Tabla 6. Coeficientes

\begin{tabular}{llccccc}
\hline \multirow{2}{*}{ Modelo } & \multicolumn{2}{c}{$\begin{array}{c}\text { Coeficientes no } \\
\text { estandarizados }\end{array}$} & $\begin{array}{c}\text { Coeficientes } \\
\text { tipificados }\end{array}$ & \multirow{2}{*}{$\mathrm{t}$} & $\mathrm{p}$ \\
\cline { 2 - 7 } & & $\mathrm{B}$ & Error típico & Beta & & \\
\hline \multirow{2}{*}{1} & (Constante) & 17,178 & 1,04 & & 16,546 & 0,00 \\
\cline { 2 - 7 } & motivaciónEX & 0,752 & 0,119 & 0,415 & 6,342 & 0,00 \\
\hline
\end{tabular}

a. Variable dependiente: Ansiedad

La Tabla 6 muestra que el valor de la constante (ansiedad) es de 17,178 cuando la variable independiente (motivación extrínseca) es igual a cero. El incremento de una unidad de la variable independiente, es decir, de la motivación extrínseca, representa el incremento de 0,752 en la escala de ansiedad. La Tabla 7 muestra la varianza de la ansiedad que puede explicarse por la variación en la motivación extrínseca.

Tabla 7. Resumen del modelo

\begin{tabular}{lccc}
\hline Modelo & R & R cuadrado & R cuadrado corregido \\
\hline 1 & $0,415^{\text {a }}$ & 0,172 & 0,168 \\
\hline
\end{tabular}

a. Variable predictiva: (Constante), motivaciónEX

Se indica en la Tabla 7 que el coeficiente de determinación corregido obtenido es de 0,168 cuando la variable predictiva es la motivación extrínseca. Eso significa que el $16 \%$ de los cambios observados en la ansiedad escolar de los niños se explica por las variaciones en la motivación extrínseca de esos niños. Además, el valor de F es de 40,221, el g es de 193 y el nivel de significancia es $p<0,001$, lo que indica que la variabilidad de la ansiedad en función de la motivación extrínseca no se debe al azar.

\section{Discusión}

El objetivo de esta investigación fue evaluar la motivación académica y la ansiedad escolar y realizar estudios de correlación y de regresión entre los constructos motivación y ansiedad. Para los análisis se decidió por la división de la motivación en motivación general, intrínseca y extrínseca, pues los dos tipos de motivación están relacionados con aspectos conductuales y afectivos diferentes.

Los resultados relativos a las puntuaciones de los chicos y las chicas mostraron que no hubo diferencia significativa en la ansiedad, no corroborando con estudios previos, como los de Silverman y cols. (1995),
Singh y cols. (2000), Batista y Oliveira (2005) y más recientemente Isolan y cols. (2011), que encontraron un promedio de los escores más elevado para el sexo femenino que para el masculino.

Es importante resaltar que las investigaciones de Silverman y cols. (1995), Singh y cols (2000), Batista y Oliveira (2005) e Isolan y cols. (2011) no se referían específicamente al contexto escolar, tal como el presente estudio, lo que podría explicar las diferencias de los resultados entre los chicos y las chicas. Como en estudios que evalúan la ansiedad general se observa constantemente un nivel superior de ansiedad de las chicas respecto a los varones, pero en esta investigación que evalúa específicamente la ansiedad en el contexto académico no se detectó diferencias entre sexo, podría plantearse que los niveles superiores de ansiedad de las chicas se deben a condiciones de otros contextos y no a las situaciones escolares.

Se resalta, asimismo, que Isolan y cols. (2011) encontraron niveles de ansiedad de moderado a alto, mientras que el presente estudio mostró que la ansiedad de los participantes no alcanzó el punto medio de la escala. Nuevamente, esa discrepancia pudo deberse a las diferentes medidas evaluadas por los instrumentos de las investigaciones, una de 
ansiedad general y la otra específica de la situación escolar.

En lo que respecta a la evaluación de la motivación, la medida de motivación general no se diferenció significativamente en función del sexo, pero sí las medidas de motivación intrínseca y extrínseca. Las chicas se mostraron más motivadas intrínsecamente en comparación con los chicos, lo que implica que ellas son más persistentes, no desisten de la adversidad de la tarea, poseen más interés en aprender, se involucran cognitivamente en las tareas y presentan más emociones positivas. En contrapartida, esta investigación reveló que los chicos son más motivados en relación a los factores extrínsecos de la tarea que las chicas, o sea, se motivan más por factores como el reconocimiento social, notas altas, ganancias materiales como recompensa. Esos datos no corroboran la información presentada en el manual por los autores del instrumento (Martinelli \& Sisto, 2011), pues en él se relatan que las diferencias entre sexo no alcanzaron la significancia estadística en las tres medidas de motivación.

El estudio comparativo de la motivación entre los varios cursos mostró que los niños de segundo curso son más motivados intrínsecamente que los niños de tercer curso. Hay un ligero incremento de la motivación intrínseca, pese a que no lega a ser significativo, en los alumnos de cuarto y quinto cursos, en comparación con los de tercero. Por ello, hay un patrón de motivación ascendiente de tercero, cuarto y quinto curso, lo que diverge de los resultados encontrados por Martinelli y Sisto (2010). Esos autores observaron una reducción de la motivación intrínseca en alumnos de cuarto y quinto curso respecto a los de tercero.

En lo que respecta a la motivación extrínseca, este estudio mostró que alumnos de tercer curso son los más motivados en relación a los factores externos al aprendizaje, seguidos de alumnos de segundo, cuarto y quinto curso. Es interesante que las medidas de motivación extrínseca no alcanzaron la media de la escala, ni para los alumnos más motivados de segundo curso. Martinelli y Sisto (2010) también observaron que niños de tercer curso mostraron una motivación extrínseca superior a los niños de cuarto y quinto, que no se diferenciaron entre sí.

Las medidas de motivación general y extrínseca mostraron una correlación significativa, positiva y de baja a moderada con la ansiedad. Esto implica que conforme hay un incremento de la necesidad de reconocimiento social, notas altas o recompensas por parte de los niños, aumentan también los síntomas y la intensidad de la ansiedad. Sin embargo, cuando los niños son motivados por la propia tarea 0 por el aprender y por la adquisición de conocimientos hay una reducción de la ansiedad escolar, reflejada por la correlación significativa, negativa y baja entre la ansiedad y la motivación intrínseca observada en este estudio, y en los trabajos de G ottfried (1985) y Guay y cols. (2008). Es importante comentar, asimismo, que la correlación negativa entre los dos tipos de motivación indica que conforme aumentan los intereses por la realización de la tarea 0 por el aprendizaje, hay una reducción de los intereses relativos a los factores externos como el reconocimiento social y las notas.

Igualmente, el análisis de regresión mostró que la motivación extrínseca fue la variable que explicó significativamente cierta varianza de la ansiedad, es decir, que posee cierto valor predictivo. Eso significa que los niños manifiestan ciertos síntomas o niveles de ansiedad que se intensifican según aumenta su motivación por sacar notas altas, recibir recompensas o por ser socialmente reconocidos.

Por lo tanto, la compilación de estudios relacionados con la motivación revela que la motivación intrínseca es importante para obtener rendimientos académicos superiores (Martinelli \& Genari, 2009; Zenorini, Santos \& Monteiro, 2011) y para mantener el equilibrio de aspectos emocionales como la ansiedad, tal como fue averiguado en este estudio y otros (Gottfried, 1985; Guay \& cols., 2008). En contraposición, la motivación extrínseca se relaciona con un rendimiento académico inferior (Zenorini \& cols., 2011) y con más síntomas de ansiedad, según revelado por este y otros trabajos (Guay \& cols., 2008). En ese sentido, por más que autores como Pintrich (2003) consideren que ambos tipos de motivación son necesarios para un buen rendimiento académico y que existan demandas reales que obliguen a las personas a preocuparse por factores externos como notas o puntuaciones en exámenes, los datos indican que cada tipo de motivación parece estar acompañado de determinadas implicaciones cognitivas y emocionales positivas y negativas.

\section{Consideraciones finales}

Este estudio presenta un pequeño avanzo en la comprensión de la relación entre la ansiedad escolar y la motivación académica. Se mostró que la ansiedad de los niños se explica en parte por las motivaciones que direccionan sus conductas, es decir, la necesidad de reconocimiento social o de sacar notas altas predice cierto incremento en el nivel de ansiedad de los niños. Sin embargo, los niños y las niñas motivados por el aprendizaje y por el desafío cognitivo de las 
actividades presentan una reducción de los síntomas de ansiedad.

No obstante, todavía quedan muchas cuestiones por averiguar, como las discrepancias encontradas en distintos estudios relacionadas con los niveles de ansiedad y el tipo de motivación que mueve a los chicos y las chicas, además de las diferencias observadas en los estudios citados relativas a la motivación y el curso de los alumnos, que podrían indicar si los niños se motivan o desmotivan a lo largo de su experiencia escolar.

Debido a la pequeña muestra de este estudio y debido a que los alumnos pertenecen a una misma escuela, esos datos son todavía exploratorios y no se pueden generalizar. Para establecer una relación más robusta entre la ansiedad académica y los tipos de motivación es necesario ampliar la cantidad de sujetos y aumentar la variabilidad de la muestra, por ejemplo, evaluar alumnos de diferentes estatus socioeconómicos, ampliar el rango de edad de los participantes, entre otros.

\section{Referencias}

Andrade, L. H. S. G., \& Gorenstein, C. (1998). Aspectos gerais das escalas de avaliação de ansiedade. Reista de Psiquiatria Clínica, 25(6), 285290.

Batista, M. A., \& Oliveira, S. M. S. S. (2005). Sintomas de ansiedade mais comuns em adolescentes. Revista Psic, 6(2), 43-50.

Bzuneck, J. A. (2000). As crenças de auto-eficácia dos professores. Em F. F. Sisto, G. de Oliveira \& L. D . T. Fini (O rgs.), Leituras depsiclogia para fomação de professures (pp. 117-134). Petrópolis, Rio de Janeiro: Vozes.

Deci, E. L., \& Ryan, R. M. (2000). The "what" and "why" of goal pursuits: human needs and selfdetermination of behavior. Psychdogial Inquiry, 11(4), 227-268.

Fortier, M. S., Vallerand, R. J., \& Guay, F. (1995). Academic motivation and school performance; toward a structural model. Contemparary Educational Psydhdogy, 20, 257-274.

Gottfried, A. E. (1985). Academic intrinsic motivation in elementary and junior high school students. Joumal of Edurational Psychoog, 77, 631-645.

Guay, F., Ratelle, C. F., \& Chanal, J. (2008). Optimal learning in optimal contexts: the role of self- determination in education. Canadian Psyddogy, 49, 233-240.

Isolan, L., Salum, G. A., O sowski, A. T., Amaro, E., \& Manfro, G. G. (2011). Psychometric properties of the Screen for Child Anxiety Related Emotional Disorders (SCARED) in brazilian children and adolescents. Jaumal of Anxiey Disardes, 25, 741748.

Martinelli, S. C., \& Genari, C. H. M. (2009). Relações entre desempenho escolar e orientações motivacionais. EstudosdePsicdoja, 14(1), 13-21.

Martinelli, S. C., \& Sisto, F. F. (2011). Escala para avaliação da mdivação esclar infantguveril (EAME-IJ). São Paulo, São Paulo: Casa do Psicólogo.

Martinelli, S. C., \& Sisto, F. F. (2010). Motivação de estudantes: um estudo com crianças do ensino fundamental. AvaliaçãoPsiclógica, 9(3), 413-420.

O liveira, S. M. S. S. (2001). Estudb exploratónio para uma scala deansiedade esdar (Dissertação de Mestrado). Universidade São Francisco, Itatiba, São Paulo, Brasil.

Oliveira, S. M. S. S., \& Sisto, F. F. (2002). Estudo para uma escala de ansiedade escolar para crianças. Psicdoja Escdar Educaional, Campinas, 6(1), 57-66.

Pintrich, P. R. (2003). A motivational science perspective on the role of student motivation in learning and teaching contexts. Jamal of Edurational Psydhogy, 95, 667-686.

Rasch, G. (1960). Probabilistic modds for some intelligence and attainment tests Copenhagen: Danmarks Paedagogiske Institut.

Ryan, R. M., \& Connell, J. P. (1989). Perceived locus of causality and internalization: examining reasons for acting in two domains. Jaumal of Pessmality and Social Psychdogy, 57, 746-761.

Silverman, W. K., La Greca, A. M., \& Wasserstein, S. (1995). What do children worry about? Worries and their relation to anxiety. Child Deudqumet, 66, 671-686.

Singh, K. A., Moraes, A. B. A., \& Ambrosano, G. M. (2000). Medo, ansiedade e controle relacionados ao tratamento odontológico. Pesquisa Odantdóġa Brasileira, 14(2), 131-136.

Urhahne, D., Chao, S. H., Florineth, M. L., Luttenberger, S., \& Paechter, M. (2011). Academic self-concept, learning motivation, and test anxiety 
of the underestimated student. Bitish Jamal of Edurational Psyddogy, 81, 161-177.

Vallerand, R. J., Fortier, M. S., \& Guay, F. (1997). Selfdetermination and persistence in a real-life setting: toward a motivational model of high school dropout. Jaumal of Personality and Social Psychdogy, 72, 1161-1176.

Vernon, M. D . (1973). Mativaçãohumana Petrópolis, RJ: Vozes.
Zenorini, R. P. C., Santos, A. A. A., \& Monteiro, R. M. (2011). Motivação para aprender: relação com 0 desempenho de estudantes. Paidia, 21(49), 157164.

Nota das autoras:

Este artigo apresenta os resultados do projeto de Iniciação Científica (PIVIC) orientado pela Profạ. Drª D ébora Cecilio Fernandes e desenvolvido pela discente do curso de psicologia Mariana Alves Silveira.

Sobre as autoras:

Débora Cecilio Femandes é doutora (2011) pela Universidad de Salamanca, no programa de Neuropsicología Clínica, com orientação do Dr. Gerardo Prieto e da Drª Ana Rosa Delgado. Atualmente, é docente dos cursos de Psicologia e Pedagogia na Universidade do Vale do Sapucaí (Univás).

Mariana Alves Siveira é discente do curso de Psicologia da Universidade do Vale do Sapucaí (Univás), atualmente, está cursando o segundo ano do curso e é aluna voluntária de Iniciação Científica da Prof ${ }^{\text {a }}$ Dra Débora Cecilio Fernandes.

Contato com as autoras:

Rua D om Mamede, 205 - Bairro João Paulo II - Código postal: 37550-000 - Pouso Alegre-MG, Brasil. E-mail: debora.cecilio@gmail.com 